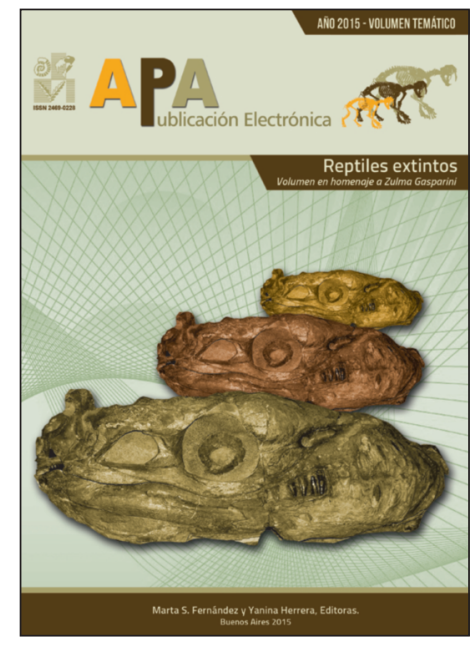

\title{
ESTADO ACTUAL DEL CONOCIMIENTO DE LOS REPTILES MARINOS CRETÁCICOS DE COLOMBIA
}

MARÍA EURÍDICE PÁRAMO FONSECA

Departamento de Geociencias, Universidad Nacional de Colombia, Carrera 30 No. 45-03, Bogotá, Colombia.

Recibido: 12 de Junio de 2015 - Aceptado: 19 de Octubre 2015

Para citar este artículo: María Eurídice Páramo Fonseca (2015). Estado actual del conocimiento de los reptiles marinos cretácicos de Colombia. En: M. Fernández y Y. Herrera (Eds.) Reptiles Extintos - Volumen en Homenaje a Zulma Gasparini. Publicación Electrónica de la Asociación Paleontológica Argentina 15(1): 40-57.

Link a este artículo: http://dx.doi.org/10.5710/PEAPA.12.06.2015.98

DESPLAZARSE HACIA ABAJO PARA ACCEDER AL ARTÍCULO

Asociación Paleontológica Argentina Maipú $6451^{\circ}$ piso, C1006ACG, Buenos Aires República Argentina

Tel/Fax (54-11) 4326-7563 Web: www.apaleontologica.org.ar

Otros artículos en Publicación Electrónica de la APA 15(1):

\section{de la Fuente \& Sterli}

ESTADO DEL CONOCIMIENTO DE LAS TORTUGAS EXTINTAS DEL TERRITORIO ARGENTINO: UNA PERSPECTIVA HISTÓRICA.

\section{Paulina Carabajal}

GUIA PARA EL ESTUDIO DE LA NEUROANATOMÍA DE DINOSAURIOS SAURISCHIA, CON ENFASIS EN FORMAS SUDAMERICANAS.

\section{Pol \& Leardi}

DIVERSITY PATTERNS OF NOTOSUCHIA (CROCODYLIFORMES, MESOEUCROCODYLIA) DURING THE CRETACEOUS OF GONDWANA. 


\title{
ESTADO ACTUAL DEL CONOCIMIENTO DE LOS REPTILES MARINOS CRETÁCICOS DE COLOMBIA
}

\author{
MARÍA EURÍDICE PÁRAMO FONSECA
}

Departamento de Geociencias, Universidad Nacional de Colombia, Carrera 30 No.45-03, Bogotá, Colombia, meparamof@unal.edu.co

\begin{abstract}
Resumen. En este artículo se pretende mostrar el estado actual del conocimiento de los reptiles marinos cretácicos de Colombia. Se ofrece una breve síntesis histórica de los estudios realizados, se brinda un panorama de los taxones presentes y se discuten algunos aspectos de su distribución geográfica y estratigráfica. La revisión realizada revela que en los sedimentos marinos cretácicos de Colombia los restos de reptiles marinos son abundantes e incluyen fósiles de tortugas, plesiosaurios, ictiosaurios y mosasaurios. Aunque las publicaciones sobre este material son aún escasas, el incremento de la participación de investigadores nacionales en el estudio de los restos de reptiles marinos ofrece un panorama alentador para el desarrollo de esta rama de la paleontología en Colombia. Los estudios y descripciones realizados ofrecen las primeras bases para sugerir que hubo cambios en la distribución, asociación y abundancia de los distintos grupos que habitaron el mar colombiano a lo largo del Cretácico. Las tortugas, los ictiosaurios y los pliosauroideos tienen una distribución limitada geográficamente y temporalmente a los inicios de la ingresión marina (Cretácico Temprano), mientras los plesiosauroideos amplían su distribución hasta el tiempo de la mayor inundación (Turoniense). Los mosasaurios aparecen con la mayor inundación y permanecen en el mar colombiano durante todo el Cretácico Tardío.
\end{abstract}

Palabras clave. Reptiles marinos. Cretácico. Colombia. Suramérica.

Abstract. STATE OF KNOWLEDGE OF THE CRETACIC MARINE REPTILES OF COLOMBIA. This article intends to show the current state of knowledge of the cretaceous marine reptiles of Colombia. A brief historical overview of the conducted studies is offered, an overview of taxa is provided and some aspects of their geographic and stratigraphic distribution are discussed. The review carried out reveals that in the Cretaceous marine sediments of Colombia the remains of marine reptiles are plentiful and include fossils of turtles, plesiosaurs, ichthyosaurs and mosasaurs. Although the literature on this material is still scarce, the increased participation of national researchers in the study of the remains of marine reptiles provides an encouraging panorama for the development of this branch of paleontology in Colombia. The studies and descriptions made offer the first bases to suggest that there were changes in the distribution, assemblage and abundance of the different groups that inhabited the Colombian sea throughout the Cretaceous. Turtles, ichthyosaurs and pliosauroids show a geographic and temporal distribution limited to the marine ingression (Early Cretaceous), while the plesiosauroids extend its distribution until the time of maximum flooding (Turonian). The mosasaurs appear during the major inundation and remain in the Colombian sea throughout the Late Cretaceous.

Key words. Marine reptiles. Cretaceous. Colombia. South America.

En Colombia se han hallado numerosos restos de reptiles marinos en rocas del Cretácico. Una reducida parte de este material ha sido publicada (Welles, 1962; Hernández-Camacho y De Porta, 1963; Huertas, 1971; de la Fuente y Goñi, 1983; Goñi y Gasparini, 1983; Hampe, 1992; Páramo, 1994; Páramo-Fonseca, 1997a, 2000, 2012, 2013; Cadena y Gaffney, 2005; Gómez-Pérez y Noè, 2009; Cadena, 2011a, b; Cadena et al., 2013) y una buena parte está actualmente en estudio. La información sobre los estudios y ocurrencia de estos restos está disgregada en notas y artículos científicos, documentos académicos, informes de prensa, co- lecciones de especímenes y comunicaciones verbales de ocurrencias, que se han reportado desde principios del siglo pasado. La excelente preservación reportada para la mayoría de los fósiles encontrados advierte del gran potencial que este material representa para el avance del conocimiento paleontológico de los reptiles marinos. Este potencial, sin embargo, se ha estudiado solo parcialmente y su magnitud aún no ha sido evaluada. En este artículo se pretende mostrar el estado actual del conocimiento de los reptiles marinos cretácicos de Colombia y ofrecer un panorama general de la distribución geográfica y estratigráfica de sus ocu- 
rrencias en el registro fósil.

Abreviaturas institucionales. CIP, Colección privada del Centro de Investigaciones Paleontológicas, Villa de Leiva, CoIombia; CFSTA, Colección privada de la Fundación Santa Teresa de Ávila, Villa de Leiva, Colombia; MJACM, Museo de la Junta de Acción Comunal de la vereda Monquirá, Villa de Leiva, Colombia; SGC, Museo Geológico José Royo y Gómez del Servicio Geológico Colombiano (anteriormente INGEOMINAS), Bogotá, Colombia; UCMP, University of California Museum of Paleontology, Berkeley, Estados Unidos; UN, Colecciones paleontológicas de la Universidad Nacional de Colombia, Villa de Leiva y Bogotá, Colombia.

\section{MATERIALES Y MÉTODOS}

Este trabajo se basó en variadas fuentes de información, la gran mayoría de ellas producto de la experiencia que ha obtenido la autora en 20 años de trabajo. La información científica seleccionada corresponde a artículos y notas publicadas en revistas científicas (e.g., Welles, 1962; Hernández-Camacho y De Porta, 1963; Huertas, 1971; de la Fuente y Goñi, 1983; Goñi y Gasparini, 1983; Hampe, 1992; Páramo, 1994; Páramo-Fonseca, 1997a, 2000, 2012, 2013; Cadena y Gaffney, 2005; Gómez-Pérez y Noè, 2009; Cadena, 2011a, b; Cadena et al., 2013), así como a trabajos y tesis académicos (Páramo-Fonseca, 1997b; Gómez-Pérez, 2001, 2008; Gómez, 2013). La información de prensa fue escogida bajo los criterios de lógica y veracidad de la información suministrada (Zárate, 2006; Moreno, 2010; Posada-Swafford, 2014; Caracol radio, 2015). Los materiales considerados en esta revisión pertenecen a colecciones oficiales y privadas. Se tienen en cuenta los reportes obtenidos en forma verbal a través de los años de trabajo en el tema. En el caso de las colecciones, la información fue depurada teniendo en cuenta las acciones de inventario adelantadas en las instituciones que las albergan. Las colecciones más representativas a este respecto son las de la Universidad Nacional de Colombia, el Servicio Geológico Colombiano y el Centro de Investigaciones Paleontológicas. Se incluyeron además las ocurrencias más destacadas de colecciones poco documentadas como la del museo "El Fósil" de Villa de Leiva y de colecciones privadas sin inventariar. En este último caso, como en el de los datos obtenidos verbalmente, la información se depuró de acuerdo con la fidelidad de las fuentes entrevis- tadas y, cuando fue posible, a través de verificaciones de campo. Existe un número no despreciable de restos fragmentarios de reptiles marinos albergados en colecciones privadas o en manos de particulares que carece de información precisa sobre su procedencia geográfica y/o estratigráfica, por lo que en este reporte sólo se mencionan si la información fue confiable o verificada en campo. En los demás casos los reportes solo se tuvieron en cuenta como testimonio de la riqueza de las localidades. Se cuentan aquí también las manifestaciones registradas en superficie que por diversas circunstancias no han llegado a excavarse.

Con el fin de visualizar la abundancia de ejemplares hallados en las distintas regiones y edades se han consignado todas las ocurrencias en las tablas ilustrativas que se citan en el texto. Para figurar la distribución estratigráfica se han tenido en cuenta sólo los hallazgos de los que se conoce una edad confiable y los mismos se han graficado sobre los esquemas de nomenclatura estratigráfica más aceptada para las distintas regiones.

\section{RESEÑA HISTÓRICA}

Los primeros reportes hechos sobre vertebrados marinos cretácicos de Colombia se limitan a menciones, principalmente de restos de peces, publicadas en trabajos e informes de reconocimiento geológico adelantados en la primera mitad del siglo XX (Hubach, 1931, 1942; Royo y Gómez, 1943; Botero-Restrepo, 1945). Hubach (1931) resalta la presencia de un nivel rico en vertebrados de edad Turoniense, extendido por la Cordillera Oriental de Colombia, al que denomina "Nivel de Peces" y del cual, en años posteriores, se extraerían importantes restos de mosasaurios. La historia del conocimiento de los reptiles marinos cretácicos de Colombia está íntimamente ligada a los hallazgos hechos en rocas del Cretácico Inferior de los alrededores de Villa de Leiva, en el departamento de Boyacá, zona central del país (Welles, 1962; Hernández-Camacho y De Porta, 1963; Huertas, 1971; de la Fuente y Goñi, 1983; Goñi y Gasparini, 1983; Hampe, 1992; Páramo-Fonseca, 1997a; Zárate, 2006; Gómez-Pérez y Noè, 2009; Moreno, 2010; PosadaSwafford, 2014; Caracol radio, 2015). Esto se debe a la abundancia de material paleontológico que contienen estas rocas, la amplia exposición superficial que exhiben estas rocas por la aridez del terreno y el fácil acceso que ofrece la 
región. Aunque el yacimiento paleontológico de Villa de Leiva ha brindado el material más abundante, no es el único en ofrecer restos de reptiles marinos cretácicos en el país. Además de este rico yacimiento, el Cretácico colombiano ha brindado fósiles de reptiles marinos en los departamentos de Huila, Tolima, Santander, Cundinamarca y Cauca (Guzmán, 1985; Páramo-Fonseca, 1997b, 2012; Cadena y Gaffney, 2005; Cadena, 2011a,b; Cadena et al., 2013).

\section{Los primeros estudios}

La primera excavación de un reptil marino cretácico realizada en Colombia se llevó a cabo hace 70 años (Fig. 1) (Botero-Restrepo, 1945). Dos esqueletos de plesiosaurios de cuello largo, casi completos y articulados, fueron extraídos en la loma La Catalina, Villa de Leiva, durante dos expediciones realizadas por el Dr. R.A. Stirton en 1945, con el apoyo de la compañía Tropical Oil Company y el Servicio Geológico Colombiano, en aquel tiempo denominado Servicio Geológico Nacional (Botero-Restrepo, 1945). Su descripción corresponde al primer estudio publicado sobre reptiles marinos de Colombia (Welles, 1962). Welles (1962) atribuye estos esqueletos a una nueva especie de elasmosaurio Alzadasaurus colombiensis y, por su excelente preservación, los toma como base para revisar todos los plesiosaurios cretácicos conocidos hasta ese momento. Alzadasaurus colombiensis es luego ubicada en el género Callawayasaurus. El holotipo, trasladado al museo de paleontología de la Universidad de California, corresponde a un esqueleto que carece tan solo de los iliones, los isquiones y las partes distales de las aletas (Welles, 1962). El segundo ejemplar, un esqueleto casi completo, reposa en las colecciones del Museo José Royo y Gómez del Servicio Geológico Colombiano, en Bogotá (Botero-Restrepo, 1945). La edad que indica Welles para estos esqueletos es Aptiense inferior con base en el estudio bioestratigráfico de Bürgl (1954). Posteriormente, en un extenso estudio bioestratigráfico sobre el Sistema Cretácico en la región de Villa de Leiva, Etayo-Serna (1968) ubica estratigráficamente la ocurrencia de reptiles marinos, entre ellos los elasmosaurios estudiados por Welles, en su denominado segmento "E" del miembro Arcillolitas Abigarradas de la Formación Paja, al cual atribuye edad Aptiense superior.

En 1963, Hernández-Camacho y De Porta describen un fragmento mandibular de la colección del Museo del Servicio Geológico Colombiano, procedente de capas del denominado "nivel de ruedas de carreta" del Coniaciense del departamento del Tolima. Los autores asignan el ejemplar a restos de ictiosaurio, mencionando con dudas su atribución a nivel familiar (Hernández-Camacho y De Porta, 1963). McGowan (1972) pone en duda que el fragmento corresponda a un ictiosaurio y Bardet (1992) propone que se trata de un fragmento mandibular de mosasaurio, siendo esta la primera vez que se mencionan restos de mosasaurios para Colombia.

El profesor y sacerdote G. Huertas, estando vinculado al Instituto de Ciencias de la Universidad Nacional de Colombia, y siendo conocedor de la gran riqueza fosilífera de Villa

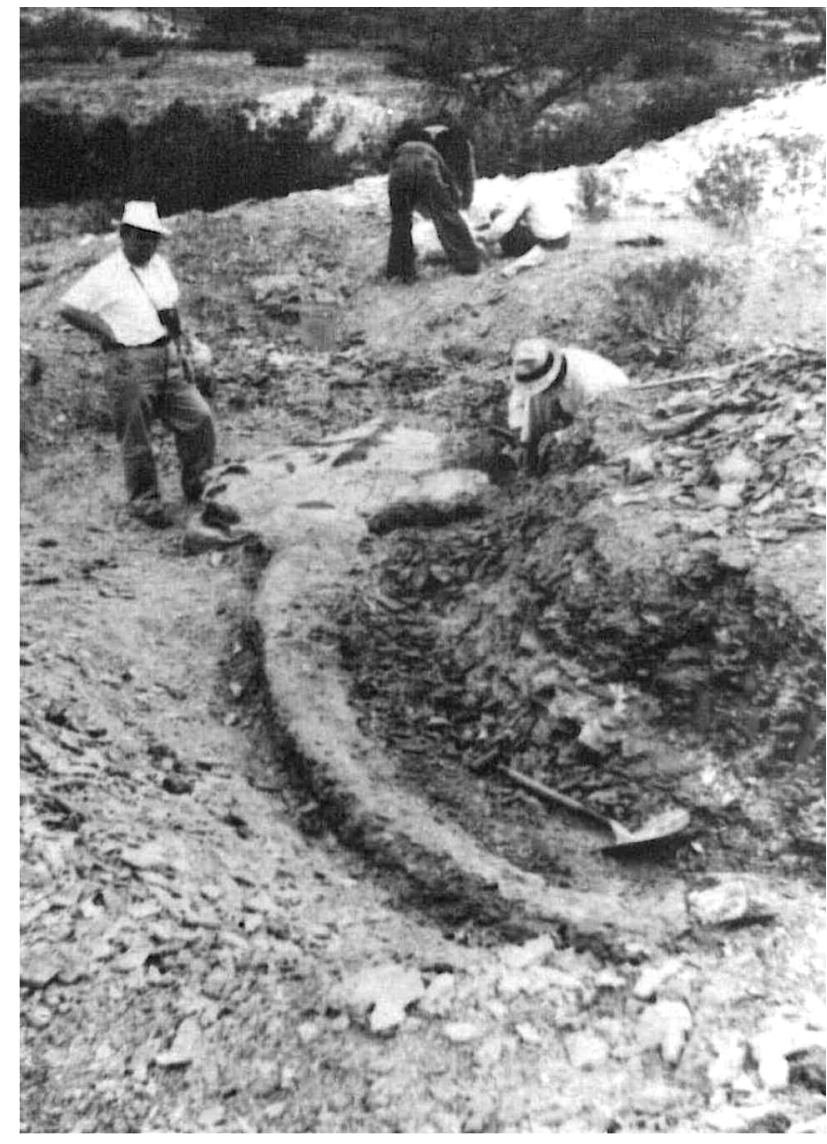

Figura 1. Excavación de Callawayasaurus colombiensis UCMP 38349 , adelantada por el Dr. R.A. Stirton en 1945. Se está destapando uno de los elasmosaurios determinados por Welles (1962). Miembro Arcillolitas Abigarradas, Formación Paja, Aptiense superior, Loma La Catalina, Villa de Leiva, Boyacá. A la izquierda el Dr. R.A. Stirton. Cortesía del Fondo documental José Royo y Gómez del Museo del Servicio Geológico Colombiano. 
de Leiva, inicia la formación del Museo Paleontológico de Villa de Leiva de esta Universidad en 1970 y dentro de sus publicaciones menciona restos de reptiles marinos (Huertas, 1971). En años posteriores, la paleontóloga argentina Zulma Gasparini visita al Padre Huertas en Villa de Leiva y se vincula por primera vez a la paleontología de Colombia a través del estudio de algunos especímenes de reptiles marinos de esta región. Como resultado de esta visita, en 1983 se publican dos estudios sobre material fósil de plesiosaurios y tortugas marinas procedentes del segmento " $E$ " de las Arcillolitas Abigarradas (Aptiense superior, Etayo-Serna, 1968) de Villa de Leiva (de la Fuente y Goñi, 1983; Goñi y Gasparini, 1983). En estas publicaciones se dan a conocer un fragmento de cráneo de Callawayasaurus colombiensis encontrado en la loma La Cabrera (Goñi y Gasparini, 1983), atribución que se encuentra hoy en revisión; y varios fragmentos craneanos de tortugas chelonioideas recolectados en la loma La Asomada (de la Fuente y Goñi, 1983).

En 1979 se publica por primera vez la presencia de restos de pliosauroideos en las rocas del Cretácico Inferior de Villa de Leiva (Acosta et al., 1979). Se trata de un esqueleto de gran tamaño, casi completo y articulado, encontrado en 1977 en rocas del Aptiense superior, que reposa hoy en su posición original y en el lugar de hallazgo, dentro de las instalaciones de un museo local en la vereda Monquirá. Acosta et al. (1979), lo asignan al género Kronosaurus, género conocido hasta ese momento solo por material fragmentario de Australia. Posteriormente Hampe (1992) publica una descripción del espécimen y lo ubica dentro de una nueva es- pecie Kronosaurus boyacensis. No obstante, para ese tiempo el material no se encontraba debidamente preparado, por lo que hoy, con una reciente preparación y a la luz de nuevos hallazgos, se pone en evidencia la necesidad de redefinir los caracteres utilizados por Hampe (op. cit.) en la definición de esta especie. Su condición articulada y casi completa hace de este espécimen un importante referente para la revisión del género Kronosaurus.

\section{La formación y los aportes de investigadores colombianos}

En 1989, durante la práctica final de campo de la carrera de Geología de la Universidad Nacional de Colombia, se halla un cráneo de mosasaurio en los alrededores de Yaguará, departamento de Huila. El espécimen se extrae de capas turonianas del Grupo Villeta, las cuales corresponden al nivel que Hubach (1931) había denominado "Nivel de Peces". Siendo estudiante de geología, la autora se involucra en la paleontología realizando el estudio de este cráneo como tesis de pregrado, bajo la dirección del Dr. Etayo-Serna. Invitada a codirigir esta tesis, la Dra. Zulma Gasparini se vincula por segunda vez al estudio de los reptiles marinos de Colombia como codirectora del trabajo. Como resultado de este estudio se identifica un nuevo género de mosasaurio (Páramo, 1994), representado por la especie Yaguarasaurus columbianus (Fig. 2). La excelente preservación tridimensional del material, la edad Turoniense y la presencia de rasgos primitivos tales como las proporciones y morfología de su región parietal, la posición adelantada de las narinas ex-

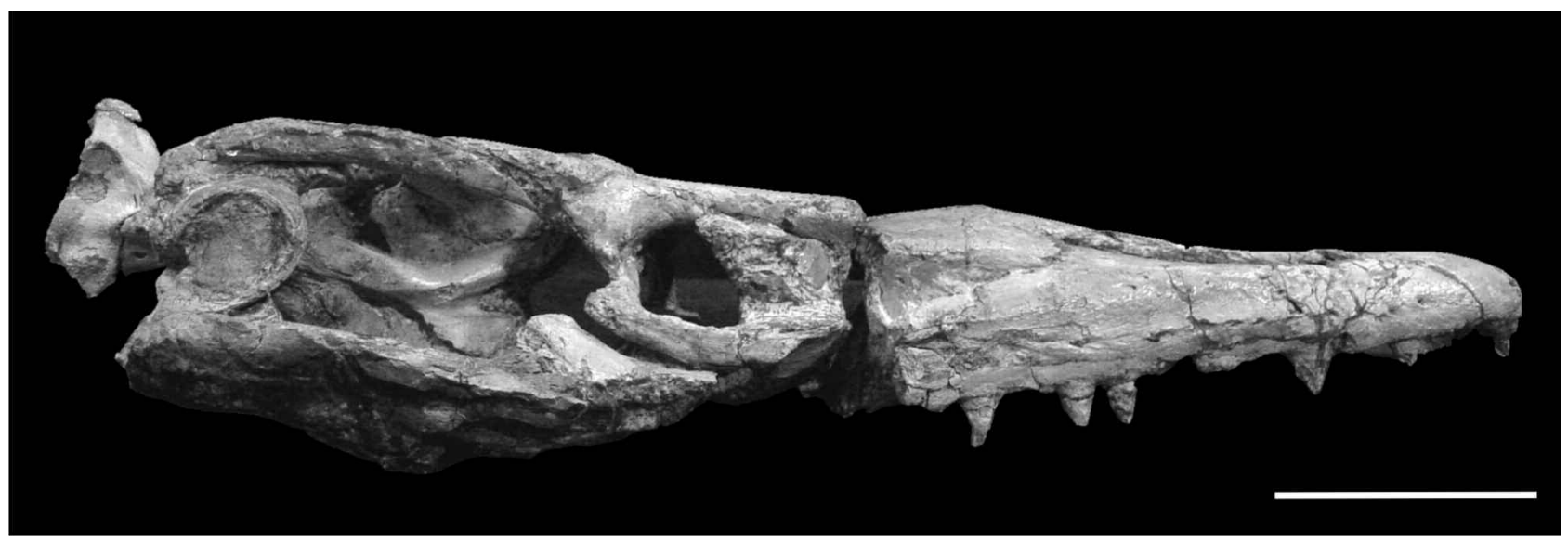

Figura 2. Yaguarasaurus columbianus UN BRV-68 (holotipo), vista lateral derecha. Escala $=10 \mathrm{~cm}$. 
ternas, el corto desarrollo del borde lingual alveolar, entre otros (ver Páramo-Fonseca, 2000), han hecho de este espécimen un referente para estudios posteriores sobre los mosasaurios tempranos (Bardet et al., 2003; Polcyn y Bell, 2005; Caldwell y Palci, 2007; Konishi y Caldwell, 2011; Palci et al., 2013).

Entre los años 1993 a 1997 los vertebrados del denominado "Nivel de Peces", en la región del Valle Superior del Magdalena (departamentos de Tolima y Huila), son estudiados por la autora en su trabajo doctoral y como resultado se identifican peces, restos fragmentarios de elasmosaurios, de mosasaurios y de otros mosasauroides procedentes del Turoniesne de esta región (Páramo-Fonseca, 1997b, 2000, 2012).

La primera mención de ictiosaurios procedentes del Cretácico Inferior de Villa de Leiva corresponde a Huertas (1971). No obstante, la primera descripción de restos de ictiosaurios es ofrecida en 1997 (Páramo-Fonseca, 1997a). En esta publicación se describe un cráneo extraído de rocas del miembro Arcillolitas Abigarradas en la loma Pedro Luis,

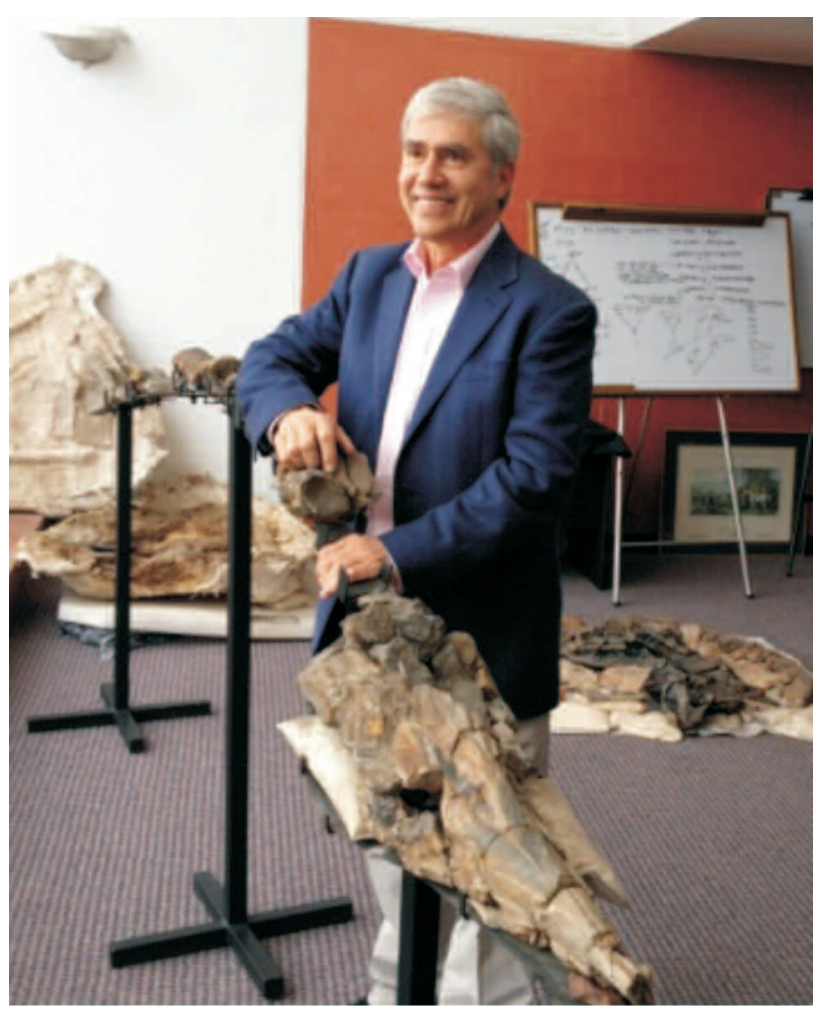

Figura 3. El Dr. C.B. Padilla junto a un ictiosaurio de su colección. Fotografía tomada en 2012. Cortesía de S. Padilla. que fue donado a las colecciones del Museo del Servicio Geológico Colombiano en la década de 1970. El espécimen, uno de los cráneos más completos conocidos para el Cretácico en ese momento, fue determinado como una especie nueva: Platypterygius sachicarum.

En 2001 se describe un segundo ejemplar de pliosaurido en una tesis de pregrado (Gómez-Pérez, 2001). Este espécimen fue encontrado por un equipo de geólogos e ingenieros y donado al Departamento de Geociencias de la Universidad Nacional de Colombia en 1967. El fósil fue haIlado en la loma La Yuca, Villa de Leiva, en capas de edad Barremiense correspondientes al Miembro Arcillolitas Abigarradas (Gómez-Pérez, 2001). El ejemplar, un cráneo casi completo y algunas vértebras cervicales, de preservación tridimensional excepcional, es identificado como Kronosaurus nov. sp. aff. K. boyacensis (Gómez-Pérez, 2001) y posteriormente determinado como un nuevo género y especie (Gómez-Pérez, 2008; Gómez-Pérez y Noè, 2009).

En 2003 el Dr. C.B. Padilla Bernal (Fig. 3), quien lideró la recolección y conservación de numerosos ejemplares de reptiles marinos de Villa de Leiva durante más de 20 años, se vincula a los estudios paleontológicos, instituyendo la Fundación Colombiana de Geobiología, con el Dr. F. EtayoSerna (Fig. 4) y la autora de este artículo como cofundadores. En los años posteriores la fundación emprende una labor continua de preparación y datación de los especímenes de la colección del Dr. Padilla y fomenta su estudio. El

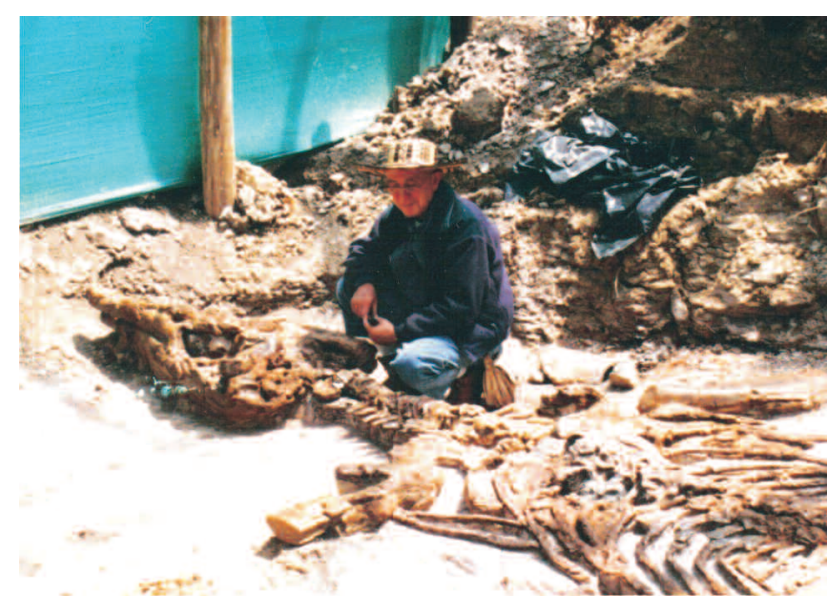

Figura 4. El Dr. F. Etayo en la excavación de un pliosaurido de las colecciones del Servicio Geológico Colombiano (SGC) realizada en 2004. Miembro Arcillolitas Abigarradas, Formación Paja, Barremiense. Loma La Catalina, Villa de Leiva, Boyacá. Cortesía del SGC. 
Dr. Padilla impulsa la formación de preparadores y promueve en Colombia la implementación de nuevas técnicas de preparación de material fósil (Padilla y Parra, 2009). Se preparan numerosos ejemplares que incluyen especímenes extraordinarios de plesiosaurios, ictiosaurios y tortugas (Páramo et al., 2009).

En 2004, en una colaboración entre el Servicio Geológico Colombiano, la Alcaldía de Villa de Leiva y la Fundación Colombiana de Geobiología, se adelanta la excavación y se inicia el estudio de un nuevo esqueleto de pliosaurido encontrado en el miembro Arcillolitas Abigarradas, en Villa de Leiva (Fig. 4). Aún sin terminar la excavación el Dr. O. Hampe visita el lugar y rápidamente publica una descripción poco precisa de este ejemplar (Hampe, 2005). Posteriormente el espécimen se incorpora a las colecciones del museo del Servicio Geológico Colombiano y una preparación química del cráneo es adelantada por el equipo técnico de la Fundación Colombiana de Geobiología. Los resultados del estudio de este espécimen permiten identificar una especie de pliosaurio diferente a las dos ya conocidas en Villa de Leiva (Páramo-Fonseca et al., en revisión), lo cual muestra para el Cretácico Inferior una diversidad del grupo hasta ahora poco documentada en el mundo.

La presencia de restos de plesiosaurios y tortugas en capas del Valanginiense de la Formación Rosa Blanca, en Zapatoca, departamento de Santander, es reportada desde 1985 por la geóloga colombiana G. Guzmán. No obstante, no es hasta 2005 que se publica el primer estudio sobre tortugas procedentes de estas capas. El geólogo colombiano E. Cadena se vincula a la paleontología recolectando nuevo material y estudiando un caparazón parcialmente completo de tortuga pleurodira (Cadena y Gaffney, 2005). En su publicación se define una nueva especie: Notoemys zapatocaensis. En estudios posteriores Cadena da a conocer nuevos restos de tortugas procedentes de la misma unidad en Zapatoca, esta vez restos fragmentarios de caparazones de pleurodiras podocnemidioideas (Cadena, 2011a) y de cryptodiras (Cadena, 2011b), además de dos caparazones incompletos de N. zapatocaensis (Cadena et al., 2013).

Con los avances alcanzados en las técnicas de preparación, la Fundación Colombiana de Geobiología implementa y publica en 2010 el uso del ácido sulfámico en la limpieza de reptiles marinos de Villa de Leiva (Padilla et al., 2010).
Luego del lamentable fallecimiento del Dr. Padilla en 2012, se liquida la Fundación Colombiana de Geobiología y la colección pasa al Centro de Investigaciones Paleontológicas (CIP) de Villa de Leiva, entidad de carácter privado.

En 2012 se dan a conocer dos nuevos hallazgos de mosasaurios (Páramo-Fonseca, 2012). En uno de los ejemplares, procedente de capas de edad Campaniense del municipio de Coello, departamento del Tolima, se conoce por primera vez el esqueleto postcraneano de un mosasaurio colombiano. En su estudio se nomina la nueva especie Eonatator coellensis. Este espécimen muestra un excelente estado de preservación, se trata de un esqueleto prácticamente completo y articulado que presenta evidencias de preservación de tejidos blandos y de restos de posibles crías (PáramoFonseca, 2013).

Entre 2012-2013 se prepara y se estudia un nuevo ejemplar de pliosaurio encontrado en la Formación Paja, en Villa de Leiva (Gómez, 2013). El ejemplar corresponde a la parte posterior de un esqueleto articulado de un individuo sub-adulto. Dentro de los pliosaurios encontrados hasta entonces en Villa de Leiva, este es el primer espécimen en el que se conoce el esqueleto caudal.

Actualmente, con la participación de estudiantes, se adelantan numerosos estudios sobre los reptiles marinos

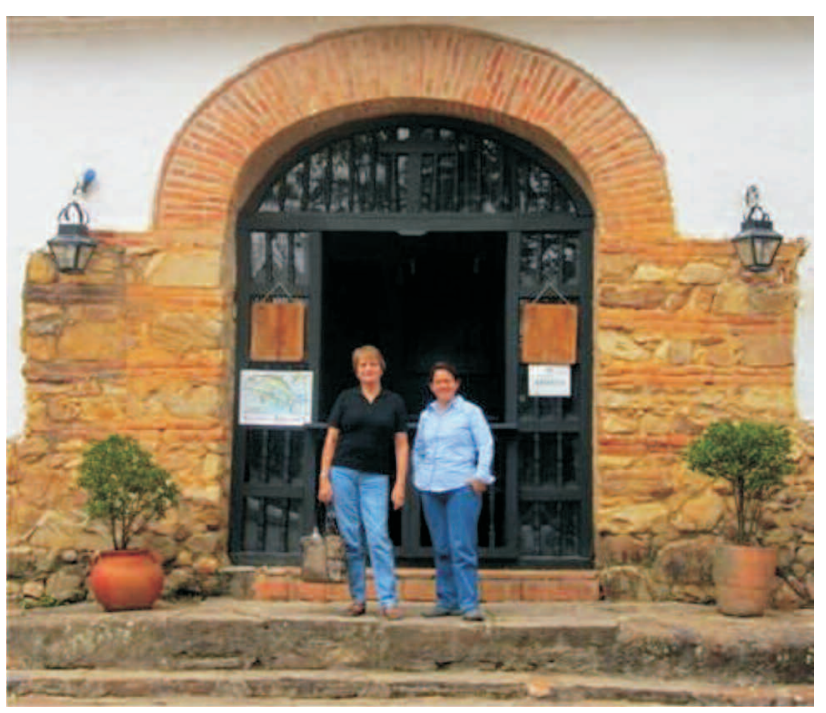

Figura 5. La Dra. Zulma Gasparini (izquierda) en su visita a Colombia en 2012, acompañada de la autora, en la puerta del Museo Paleontológico de la Universidad Nacional de Colombia en Villa de Leiva. Cortesía de C. Gasparini. 
del Cretácico de Colombia. Aunque se trabaja en los mosasaurios de los Departamentos de Santander y del Huila, la mayor parte de los estudios que se desarrollan actualmente involucran los fósiles de ictiosaurios, tortugas y plesiosaurios del Cretácico Inferior de Villa de Leiva de la colección del CIP. En el estudio de los ictiosaurios se ha vinculado recientemente a la comunidad universitaria de la región de Villa de Leiva; en el estudio de las tortugas se ha comenzado a publicar resultados (Cadena y Parham, 2015); y en el estudio de los plesiosaurios se trabaja con la participación de la Dra. Zulma Gasparini quien de esta manera se ha vinculado por tercera vez a la paleontología de los reptiles marinos de Colombia (Fig. 5).

\section{OCURRENCIAS DE REPTILES MARINOS CRETÁCICOS EN COLOMBIA}

Con base en la información recopilada, se presenta aquí un panorama de las ocurrencias de restos de reptiles marinos en las capas del Cretácico de Colombia. Se sintetiza la información señalada en las publicaciones y se menciona el material de acceso público resguardado en colecciones oficiales y privadas.

La localización geográfica de los sitios de hallazgos se ilustra en la Figura 6. El nivel estratigráfico de los mismos se sintetiza en las Figuras 7-10. En estas figuras se muestran las ocurrencias de reptiles marinos de acuerdo con la nomenclatura estratigráfica utilizada en las distintas regiones geológicas. Estas figuras corresponden de norte a sur a San-

TABLA 1. Abundancia de ocurrencias de reptiles marinos en Colombia a través del Cretácico.

\begin{tabular}{|c|c|c|c|c|c|c|c|c|c|c|c|}
\hline & \multicolumn{4}{|c|}{ Testudines } & \multicolumn{2}{|c|}{ Ichthyosauria } & \multicolumn{3}{|c|}{ Plesiosauria } & \multicolumn{2}{|c|}{ Mosasauroidea } \\
\hline & $\begin{array}{l}\frac{8}{0} \\
\frac{0}{2} \\
\frac{d}{5} \\
\frac{0}{2} \\
\frac{0}{2}\end{array}$ & 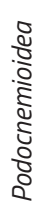 & 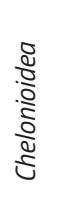 & 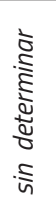 & 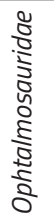 & 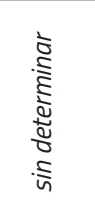 & 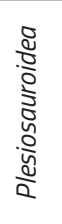 & 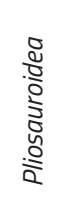 & 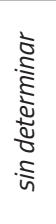 & 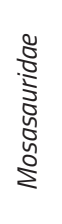 & 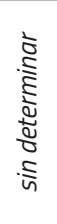 \\
\hline Maastrichtiense & & & & $x(?)$ & & & & & & & \\
\hline Campaniense & & & & & & & & & & $x$ & \\
\hline \multicolumn{12}{|l|}{ Santoniense } \\
\hline Coniaciense & & & & & & & & & & $x x$ & \\
\hline Turoniense & & & & & & & $x x$ & & & $x x$ & $x$ \\
\hline \multicolumn{12}{|l|}{ Cenomaniense } \\
\hline Albiense & & & & & & $x$ & & & & & \\
\hline \multicolumn{12}{|l|}{ Aptiense } \\
\hline & & & $x x x$ & $x x x$ & $x x$ & $x X X X X X$ & $x x x$ & $x x x x$ & $x x$ & & \\
\hline \multicolumn{12}{|l|}{ Barremiense } \\
\hline \multicolumn{12}{|l|}{ Hauteriviense } \\
\hline Valanginiense & $x x$ & $x x$ & & $x$ & & $x$ & $x$ & & & & \\
\hline Berriasiense & & & & & & & & & & & \\
\hline
\end{tabular}

Número de ejemplares: $x: 1 ; x x: 2-5 ; x x x: 6-10 ; x x x x: 11-15 ; x x x x x: 16-20 ; x x x x x x:$ más de 20.

Figura 6. Distribución geográfica de la ocurrencia de reptiles marinos cretácicos en Colombia. Mapa físico de la República de Colombia a escala 1: 3.000.000, con relieve, Instituto Agustín Codazzi, 1995. Localidades: Lebrija (1); Zapatoca (2); Galán (3); Villa de Leiva, Sáchica y Sutamarchán (4); Guayabal de Síquima (5); Bojacá (6); Piedras (7); Coello (8); Cunday (9); Dolores (10); Yaguará (11); Itaibe (12). Escala= 150 km. 


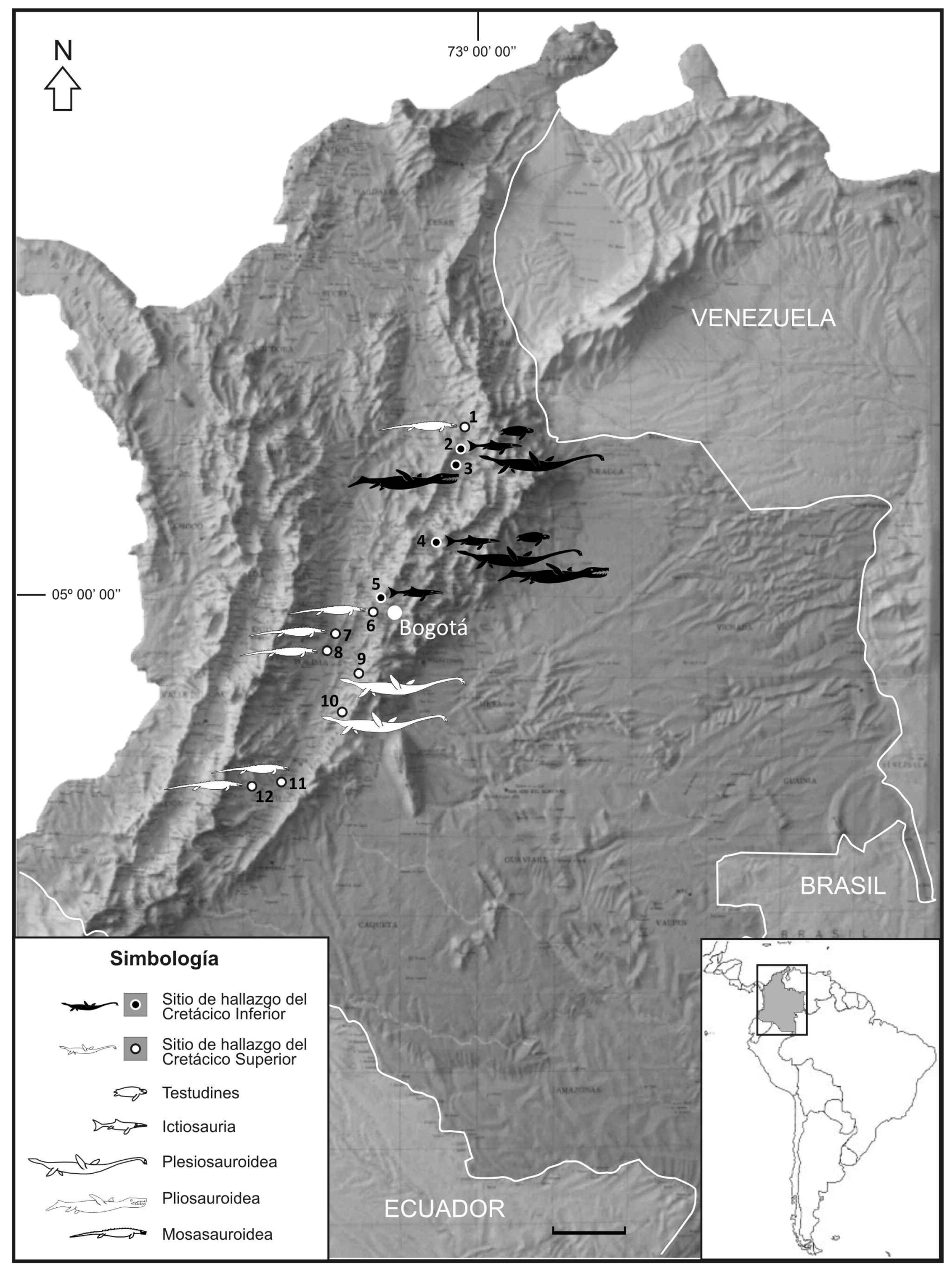


tander (localidades 1-3, Fig. 6); Villa de Leiva (localidad 4, Fig. 6); Cundinamarca y Tolima (localidades 6-10, Fig. 6); y Huila y Cauca (localidades 11-12, Fig. 6). Para la región de Villa de Leiva (Fig. 8) la gráfica se basó solo en los especímenes de los que se cuenta con información suficiente para establecer si provienen del piso Barremiense o del Aptiense.

A continuación se enumeran las ocurrencias de reptiles marinos cretácicos de Colombia organizadas por grupos taxonómicos. Con el fin de ofrecer una idea de la abundancia relativa de especímenes con respecto a la edad y la localidad, en las Tablas 1 y 2 se representa de manera resumida la ocurrencia general de hallazgos.

\section{TESTUDINES Linnaeus, 1758}

Varios restos fragmentarios de caparazones (UN 36234, UN 3638b/18), Valanginiense, Formación Rosa Blanca, Zapatoca (Santander).

Huevos y restos poscraneanos (UN sin número de colección; Etayo-Serna, com. pers.), varios esqueletos parciales, con y sin cráneo (CIP FCG-CBP-14, CIP FCG-CBP-20, CIP FCG-CBP-31); restos fragmentarios (MJACM, sin número de colección), Barremiense-Aptiense, Miembro Arcillolitas Abigarradas, Formación Paja, Villa de Leiva (Boyacá) (Páramo et al., 2009); restos fragmentarios (UN MPVL 63), Barremiense, Miembro Arcillolitas Abigarradas, Formación Paja, Villa de Leiva (Boyacá).

Restos fragmentarios (CIP sin número de colección), Miembro Arcillolitas Abigarradas, Formación Paja, Barremiense-Aptiense, Sáchica (Boyacá).

Cráneo y elementos poscraneanos (UCMP 38347), Maastrichtiense, departamento de Santander (UCMP, 2015). Dada la información con que se cuenta sobre ocurrencias en el departamento de Santander y la pobre información sobre la procedencia de este material, la edad de este reporte es aquí puesta en duda.

Huevos (UCMP 38348), Cretácico Inferior, departamento de Cundinamarca (?) (UCMP, 2015). En un documento de campo de 1945, W.T. O'Gara de la Tropical Oil Company reporta el hallazgo de restos óseos y probables huevos de tortuga. De la información suministrada en el documento se infiere que los fósiles mencionados provienen de Villa de Leiva, Boyacá, del Miembro Arcillolitas Abigarradas (Forma- ción Paja, Barremiense-Aptiense). Aunque el destino final de los especímenes no se clarifica en el documento sí se menciona que hubo material enviado a Berkeley, California.

Restos sin identificar (UCMP 57831), Cretácico Inferior, departamento de Tolima (UCMP, 2015).

Testudines indet.

Cráneo mal preservado (UN ICNMHNR-083) (de la Fuente y Goñi, 1983), Aptiense, Miembro Arcillolitas Abigarradas, Formación Paja, Villa de Leiva (Boyacá).

Pleurodira Cope, 1864

Platychelyidae Bräm, 1965

Notoemys zapatocaensis Cadena y Gaffney, 2005

Caparazón y parte posterior de plastrón (SGC IPN15EAC140120031, holotipo), otro caparazón casi completo y restos fragmentarios (SGC IPN15-EAC150620061), Valanginiense, Formación Rosa Blanca, Zapatoca (Santander) (Cadena y Gaffney, 2005; Cadena et al., 2013).

Podocnemidoidea Cope, 1868

Podocnemidoidea indet.

Fragmentos de caparazón (SGC IPN16EAC-140120031A, SGC IPN16EAC-14012003-1B), Valanginiense, Formación Rosa Blanca, Zapatoca (Santander) (Cadena, 2011a).

\section{CRYPTODIRA Cope, 1868}

En las colecciones del Departamento de Geociencias se encuentra un elemento de caparazón (UN 36232/4) con los rasgos ilustrados por Cadena (2011a) para el elemento costal 5 de Caretta caretta, por lo que aquí se incluye en Cryptodira. Valanginiense, Formación Rosa Blanca, Zapatoca (Santander).

\section{EUCRYPTODIRA Gaffney, 1975}

Eucryptodira gen. et sp. Indet

Restos fragmentarios de caparazones (SGC IPN15EAC14012003-4A，SGC IPN15-EAC14012003-4B， SGC 
IPN15-EAC14012003-4C, SGC IPN15-EAC14012003-4D), Valanginiense, Formación Rosa Blanca, Zapatoca (Santander) (Cadena, 2011b).

\section{Chelonioidea Baur, 1893}

Protostegidae Cope, 1872

Desmatochelys Williston, 1894

Desmatochelys padillai Cadena y Parham, 2015

Un esqueleto incompleto con cráneo (CIP FCG-CBP 01, holotipo) y varios cráneos (CIP FCG-CBP 13, CIP FCG-CBP 15, CIP FCG-CBP 39, CIP FCG-CBP 40) (Cadena y Parham, 2015), Barremiense y Aptiense, Miembro Arcillolitas Abigarrada, Formación Paja, Villa de Leiva (Boyacá); fragmentos de caparazón (UCMP 38345A, UCMP 38345B) (Cadena y Parham, 2015); un cráneo completo y articulado (UCMP 38346) (Smith, 1989; Cadena y Parham, 2015), Smith (1989) menciona la procedencia de esta muestra con número V 4538/38346 como Cretácico Inferior de Columbia (USA); Nicholls y Meckert (2002) hacen referencia a la publicación de Smith confiriendo su procedencia al Aptiense de Colombia; en la información digital del UCMP se encuentra el número del espécimen citado por Smith (V 4538/38346) con procedencia Barremiense-Aptiense, Boyacá, Colombia; Cadena y Parham (2015) reportan la muestra, junto con las demás del UCMP y las del CIP, con edad Barremiense superior-Aptiense inferior, sin diferenciar.

Chelonildae Gray, 1825

Cheloniidae?

Una mandíbula (UN ICNMHNR-082), Aptiense superior, Miembro Arcillolitas Abigarradas, Formación Paja, Villa de Leiva (Boyacá) (de la Fuente y Goñi, 1983).

ICHTHYOSAURIA de Blainville, 1835

Vértebras (Cadena, com. pers.), Valanginiense (?), Formación Rosa Blanca, Zapatoca, Santander.

Restos vertebrales (UN MP 110210-7, UN MPVL-91026, UN MPVL-91-028, UN MPVL-91-031, UN MPVL-91059, UN MPVL-91-062, UN MPVL-91-063, UN MPVL 127); cráneos, entre completos y parciales y con otros restos postcraneanos (UN MPVL-91-064; CIP FCG-CBP-5, CIP FCG-CBP-8, CIP FCG-CBP-10, CIP FCG-CBP-12, CIP FCGCBP-18, CIP FCG-CBP-19, CIP FCG-CBP-23, CIP FCG-CBP27, CIP FCG-CBP-32, CIP FCG-CBP-33, CIP FCG-CBP-63, CIP FCG-CBP-70, CIP FCG-CBP-72, CIP FCG-CBP-87), gran parte de este material se encuentra en estudio; restos fragmentarios (MJACM 3, MJACM 11), Barremiense y Aptiense, Miembro Arcillolitas Abigarradas, Formación Paja, Villa de Leiva (Boyacá).

Restos fragmentarios (CIP FCB-CBP-30), Aptiense, Miembro Arcillolitas Abigarradas, Formación Paja, Sáchica (Boyacá).

Vértebras y costillas (SGC RB-102011), Albiense, Grupo Villeta Guayabal de Síquima (Cundinamarca).

Ophthalmosauridae Baur, 1887

Cráneo y columna vertebral casi completa (CIP FCG(BP-16), Aptiense, Miembro Arcillolitas Abigarradas, Formación Paja, Villa de Leiva (Boyacá) (García y Páramo, en preparación).

Platypterygius sachicarum Páramo-Fonseca, 1997a

Un cráneo incompleto (SGC DON-19671 holotipo), Barremiense-Aptiense, Miembro Arcillolitas Abigarradas, Formación Paja, Villa de Leiva (Boyacá) (Páramo-Fonseca, 1997a).

Plesiosauria de Blainville, 1835

Fragmentos de miembros (UN MPVL-91-015, UN MPVL-91-016, UN MPVL-91-041), Barremiense-Aptiense, Miembro Arcillolitas Abigarradas, Formación Paja, Villa de Leiva (Boyacá).

\section{Plesiosauroidea Welles, 1943 \\ ElasmosauridaE Cope, 1869}

Vértebras desarticuladas (UN 36377, UN 36330, UN 36219), Valanginiense, Formación Rosa Blanca, Zapatoca (Santander) (Guzmán, 1985). 


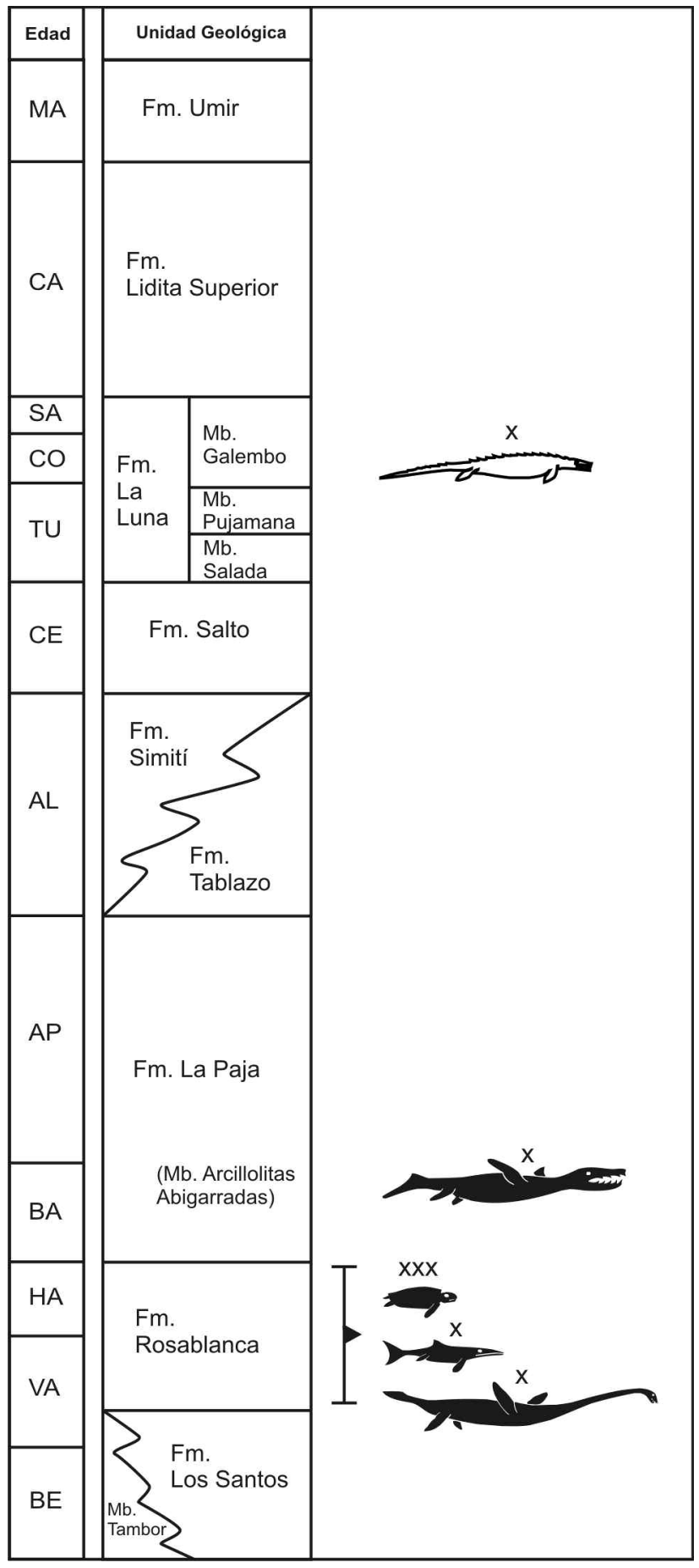

Figura 7. Localización estratigráfica de los hallazgos hechos en el departamento de Santander. Nomenclatura estratigráfica basada en el Proyecto Sogamoso desarrollado por el Servicio Geológico Colombiano (SGC) (en prep.). Cortesía del SGC. Simbología como en figura 5. Número de ejemplares: $\mathbf{x}, 1 ; \mathbf{x x}$, entre $2-5 ; \mathbf{x x x}$, entre $6-10$.
Vértebras (UN MPVL-91-024, UN MPVL-91-025, UN MPVL-91-029, UN MPVL-91-033, UN MPVL-91-034, UN MPVL-91-038; CIP FCG-CBP-24), parte anterior de un esqueleto articulado y fragmento de cráneo (CIP FCG-CBP-3, CIP FCG-CBP-22), Barremiense y Aptiense, Miembro ArciIlolitas Abigarradas, Formación Paja, Villa de Leiva (Boyacá) (Páramo-Fonseca et al., en preparación).

Vértebras cervicales y dorsales y fragmentos mandibulares (material en préstamo al SGC, sin número de colección), Turoniense, Formación La Frontera, Cunday (Tolima).

\section{Elasmosauridae indet.}

Fragmentos vertebrales (SGC DON1995-12). Turoniense superior, Grupo Villeta, Dolores (Tolima) (Páramo-Fonseca, 1997b).

Callawayasaurus colombiensis (Welles, 1962) Carpenter, 1999

Cráneo y esqueleto incompleto articulado (UCMP 38349 holotipo) y cráneo incompleto (paratipo) (UCMP 125328), esqueleto casi completo articulado sin cráneo (paratipo) (SGC sin número de colección) (Welles, 1962; Etayo-Serna, 1968; Carpenter, 1999); parte anterior de un cráneo (UN ICNMHNR-081) (Goñi y Gasparini, 1983), Aptiense superior, Miembro Arcillolitas Abigarradas, Formación Paja, Villa de Leiva (Boyacá). Al parecer el esqueleto (paratipo) del SGC y el cráneo incompleto (paratipo) de las colecciones de UCMP conforman un mismo ejemplar.

\section{Pliosauroidea Welles, 1943}

Dos esqueletos casi completos (SGC IPN-38; CIP FCGCBP-4), restos craneanos (CIP FCG-CBP-4, CIP FCG-CBP-6, CIP FCG-CBP-7, CIP FCG-CBP-9, CIP FCG-CBP-26, CIP FCGCBP-29; CFSTA 2-1; MJACM 2), restos fragmentarios postcraneanos (MJACM 5), Barremiense y Aptiense, Miembro Arcillolitas Abigarradas, Formación Paja, Villa de Leiva (Boyacá).

Un esqueleto con cráneo y sin aletas (UN MP11209-1) y un cráneo completo (UN MP050310-1) (Gutiérrez y Páramo-Fonseca, en preparación), un cráneo completo (CIP 
FCG-CBP-21), Barremiense-Aptiense, Miembro Arcillolitas Abigarradas, Formación Paja, Sáchica (Boyacá).

Un cráneo (SGC sin número de colección), BarremienseAptiense, Formación Paja, Galán (Santander).

\section{Pliosauridae Seeley, 1874}

Un esqueleto casi completo (SGC VL17052004-1), Barremiense, Miembro Arcillolitas Abigarradas, Formación Paja, Villa de Leiva (Boyacá) (Páramo-Fonseca et al., en revisión).

Cráneo casi completo y vértebras cervicales (UN UN-DGR-1000) (Gómez-Pérez, 2008; Gómez-Pérez y Noè, 2009; Gómez-Pérez, en revisión), Barremiense, Miembro Arcillolitas Abigarradas, Formación Paja, Sutamarchán (Boyacá).

Kronosaurus boyacensis Hampe, 1992

Esqueleto casi completo articulado (MJACM 1, holotipo), Aptiense superior, Miembro Arcillolitas Abigarradas, Formación Paja, Villa de Leiva (Boyacá) (Hampe, 1992).

\section{Pliosauridae aff. Kronosaurus boyacensis}

Parte posterior de un esqueleto (UN MP110210-1), Barremiense, Miembro Arcillolitas Abigarradas, Formación Paja, Villa de Leiva (Boyacá) (Gómez-Pérez, 2013).

Mosasauroidea Camp, 1923

Una vértebra (SGC MP-13), Turoniense, Grupo Villeta, Yaguará (Huila) (Páramo-Fonseca, 1997a, 2012).

MosASAURIDAE Gervais, 1853

Una vértebra dorsal (SGC MP-4), Turoniense, Grupo Villeta, Yaguará (Huila) (Páramo-Fonseca, 1997b, 2012).

Fragmento mandíbular (SGC sin número de colección),

Figura 8. Localización estratigráfica de los hallazgos hechos en la región de Villa de Leiva. Columna estratigráfica tomada y modificada de Forero y Sarmiento (1985). Simbología como en la figura 5. Número de ejemplares: $\mathbf{x}, 1$; xx, entre $2-5$; xxx, entre $6-10$; xxxx, entre 11-15.

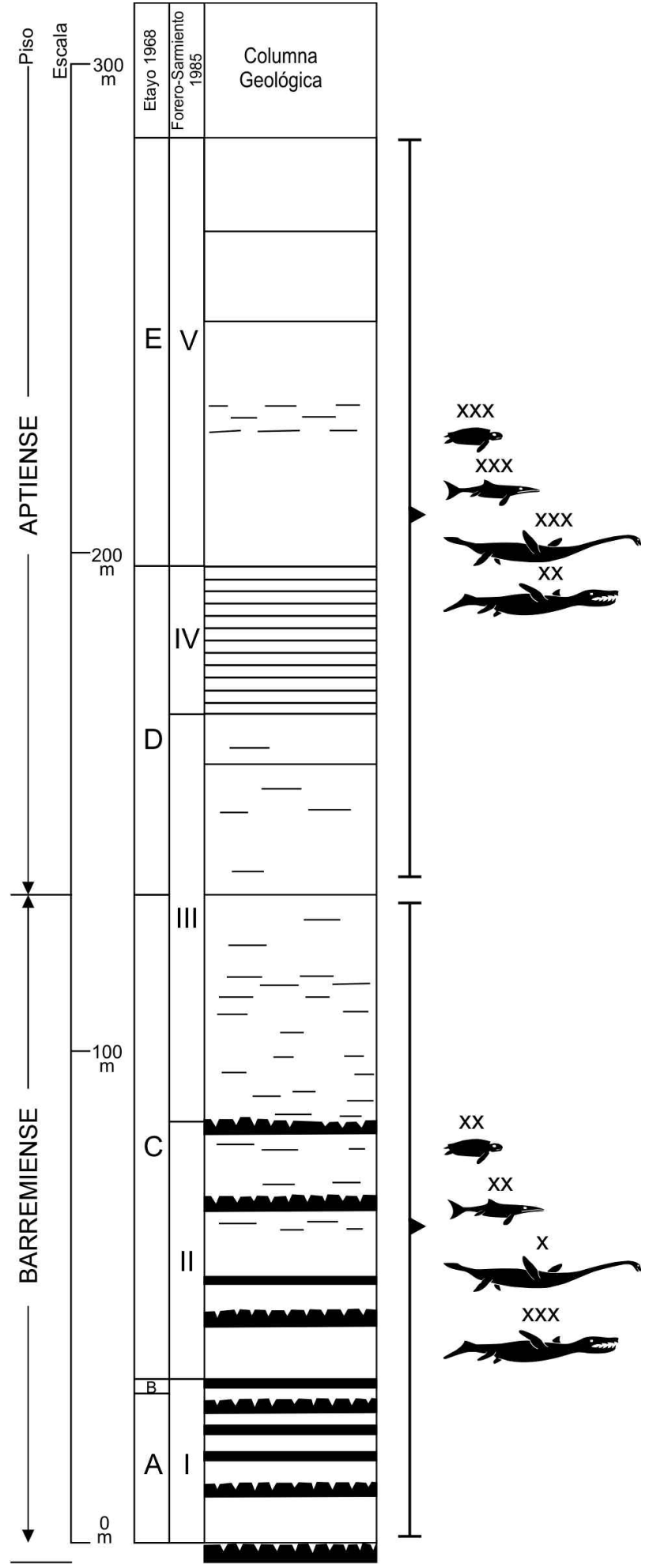

Símbolos litológicos

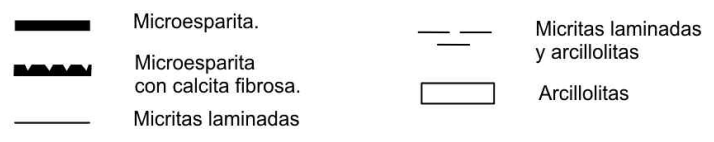


Coniaciense, Grupo Villeta, Piedras (Tolima) (HernándezCamacho y De Porta, 1963).

Un cráneo incompleto (SGC IPN-2), Coniaciense, Formación La Luna, Lebrija (Santander) (Páramo-Fonseca, 2012, en preparación).

En la colección del SGC se encuentra un molde de restos fragmentarios craneanos desarticulados (SGC OB 032001-1), Coniaciense-Santoniense, Grupo Villeta-Grupo Olini, Bojacá (Cundinamarca).

Yaguarasaurus columbianus Páramo, 1994

Un cráneo y primeras vértebras cervicales (UN BRV-68 holotipo) (Páramo, 1991, 1994), restos fragmentarios craneanos y un diente (SGC MP-1, MP-2) (Páramo-Fonseca, 1997b, 2000, 2012), Turoniense superior, Grupo Villeta, Yaguará (Huila).

Un cráneo incompleto con vértebras cervicales y dorsales (SGC MP-14), Turoniense, Grupo Villeta, Itaibe (Cauca) (Páramo-Fonseca, 1997b, 2000, 2012).

\section{Eonatator coellensis Páramo-Fonseca, 2013}

Esqueleto completo articulado (SGC IGMp881237 holotipo), Campaniense, Nivel de Lutitas y Arenas, Coello (Tolima) (Páramo-Fonseca, 2012, 2013).

\section{ESTADO ACTUAL DEL CONOCIMIENTO}

La información revisada muestra que Colombia posee una gran riqueza fósil de reptiles marinos del Cretácico, siendo la región de Villa de Leiva la más prolífica hasta ahora (Fig. 8, Tab. 2). No obstante, los estudios publicados solo cubren una parte de esta riqueza. A partir del recuento histórico se puede señalar que en la historia de las publicaciones se manifiesta un significativo aumento en la producción científica desde la década de los 90s que se relaciona con la vinculación de investigadores nacionales a la paleontología de Colombia. La formación de nuevos paleontólogos en el país se ve incentivada en la primera década del siglo XXI, con lo cual el número de las publicaciones sobre nuevos especímenes de reptiles marinos se incrementa a partir del 2010. Los aportes al conocimiento revelan un desarrollo significativo de la actividad paleontológica sobre los reptiles marinos de Colombia, cuyos resultados permiten vislumbrar por primera vez una historia dinámica en la ocupación, distribución y permanencia de los reptiles marinos en el mar cretácico de Colombia.

La presencia de fósiles de reptiles marinos en Colombia se relaciona íntimamente con la distribución de los sedimentos cretácicos que se encuentran en la Cordillera Oriental (Fig. 6). Estos sedimentos registran la ingresión y

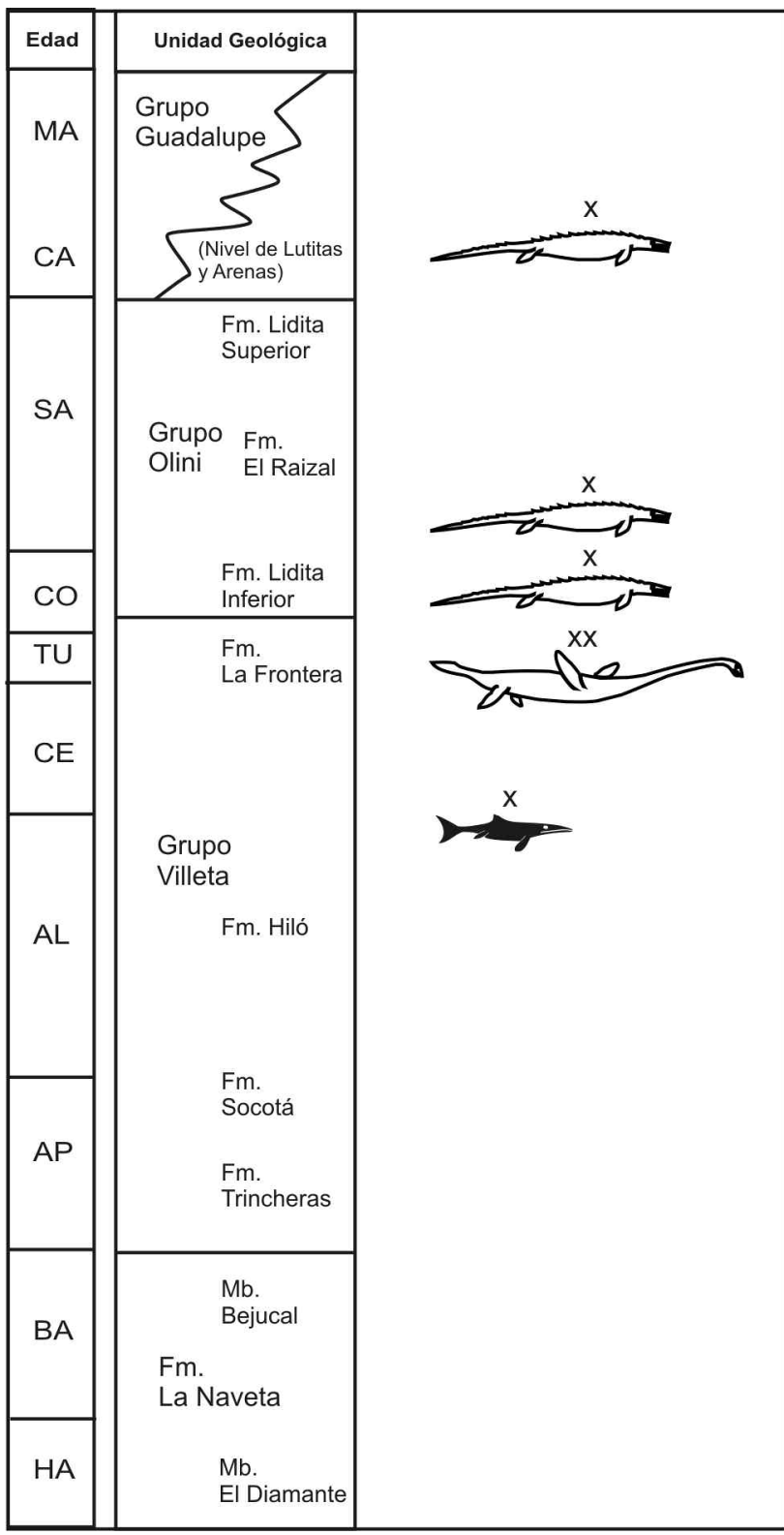

Figura 9. Localización estratigráfica de los hallazgos hechos en los departamentos de Cundinamarca y Tolima. Nomenclatura estratigráfica basada en Etayo-Serna (1979). Simbología como en la figura 5. Número de ejemplares: $\mathbf{x}, 1 ; \mathbf{x x}$, entre $2-5$. 
TABLA 2. Abundancia de ocurrencias de reptiles marinos cretácicos por localidad geográfica.

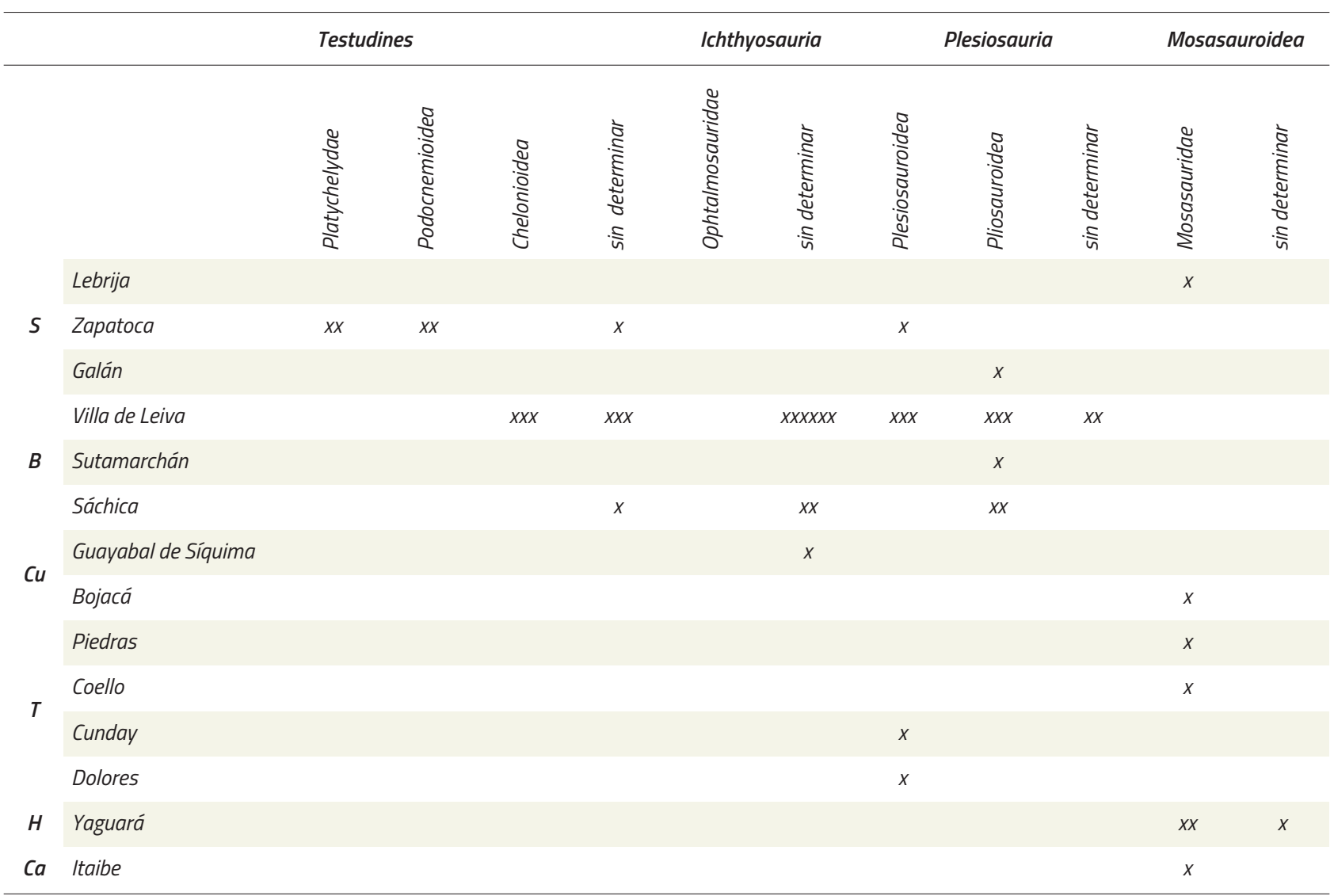

Número de ejemplares: x: 1; xx: 2-5; xxx: 6-10; xxxx: 11-15; xxxxx: 16-20; xxxxxx: más de 20. Departamentos: B: Boyacá; Ca: Cauca; Cu: Cundinamarca; H: Huila; S: Santander; T: Tolima.

posterior regresión del mar sobre la parte occidental del territorio Colombiano durante el Cretácico (Etayo-Serna et al., 1976). Los estudios adelantados sobre los reptiles marinos han incluido ocurrencias en distintos pisos del Cretácico, abarcando desde el Valanginiense hasta el Campaniense. La información sintetizada muestra que las capas del Cretácico Inferior han brindado dos yacimientos significativos. Por una parte, numerosos especímenes de tortugas y restos aislados de plesiosaurios e ictiosaurios aparecen en capas del Valanginiense en la región de Zapatoca, Santander (Fig. 7, Tabs. 1-2). Por otra parte, se cuenta con abundante material de la región de Villa de Leiva que incluye ictiosaurios, tortugas y plesiosaurios del Barremiense y del Aptiense (Fig. 8, Tabs. 1-2). El Cretácico Superior ha ofrecido varios especímenes de mosasaurios y algunos plesiosaurios en el Turoniense del Valle Superior del Magdalena (Fig. 10, Tab. 1), así como especímenes aislados de mosasaurios en el Coniaciense de la región central del país y, más al norte, en el Campaniense de Santander (Figs. 7, 9, Tab. 1).

Los fósiles de tortugas marinas del Cretácico de Colombia muestran hasta ahora una distribución limitada al Cretácico Inferior de las regiones central y norte de la Cordillera Oriental (Figs. 6-7). Su ocurrencia revela diversidad desde sus primeros registros en el Valanginiense (Tab. 2) y presencia de formas desconocidas en otros lugares del mundo, lo cual sugiere que las tortugas Ilegaron al mar cretácico de Colombia en tiempos anteriores al Valanginiense.

El registro fósil de ictiosaurios muestra una alta concentración de esqueletos en la región central de la Cordillera Oriental, depositados durante el Barremiense y en mayor número durante el Aptiense (Figs. 6-8, Tab. 2). Los especímenes presentan variada morfología lo que sugiere que 


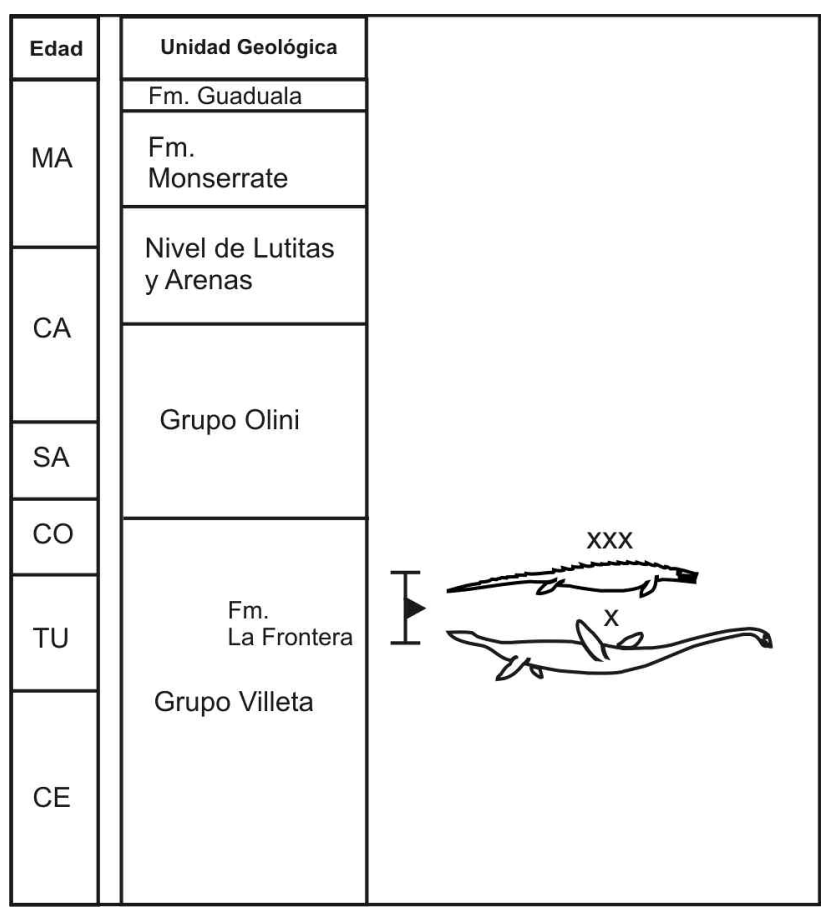

Figura 10. Localización estratigráfica de los hallazgos hechos en los departamentos de Huila y Cauca. Nomenclatura estratigráfica basada en Etayo-Serna (1994). Simbología como en la figura 5. Número de ejemplares: $\mathbf{x}, 1$; xx, entre $2-5$; xxx , entre $6-10$.

hubo una gran proliferación y alta diversidad de ictiosaurios durante el Cretácico Inferior en el mar colombiano. La presencia de restos en el Albiense (Fig. 9) revela que los ictiosaurios estuvieron presentes en el mar cretácico de Colombia hasta los finales de su historia.

Los plesiosauroideos están registrados en rocas que abarcan desde el Valanginiense hasta el Turoniense (Tab. 1), los más antiguos en el norte y centro de la Cordillera Oriental y los más recientes en el Valle Superior del Magdalena, en la zona central y sur del país (Figs. 6-10, Tab. 2). Por su parte, los pliosauroideos que se conocen hasta ahora están restringidos al Barremiense y al Aptiense de la zonas central y norte de esta cordillera (Figs. 6-8, Tab. 2), pero mostrando una mayor abundancia (Tab. 2) y variedad que los plesiosauroideos de estos mismos pisos. Este panorama sugiere que los plesiosaurios de cuello largo conquistaron las aguas del mar cretácico de Colombia desde los inicios de la ingresión marina y se fueron extendiendo por las nuevas áreas inundadas, mientras que los de cuello corto se instalaron en la zona central y se diversificaron rápidamente. A pesar de que la información relativa a la edad de los reptiles marinos encontrados en la región de Villa de Leiva es incompleta, es interesante observar que se percibe un aumento en la cantidad de especímenes de ictiosaurios, tortugas y plesiosauroideos hallados en el Aptiense con relación a la de los hallados en el Barremiense (Fig. 8). A diferencia de estos, los pliosauroides no muestran este aumento.

Los mosasaurios parecen estar representados a lo largo de la Cordillera Oriental (Fig. 6, Tab. 2) en niveles que incluyen casi todo el Cretácico Superior (Figs. 7, 9-10, Tab. 1), desde el Turoniense, que corresponde al tiempo en que los mosasaurios aparecieron en los mares del mundo, hasta el Campaniense, cuando se acercan al final de su historia. Esta distribución sugiere una amplia existencia y permanencia de mosasaurios en el mar cretácico de Colombia, lo cual en combinación con la excelente preservación de sus restos, representa un importante potencial de estudio para la paleontología colombiana.

Para todos los grupos, los especímenes determinados corresponden, al nivel de géneros o especies, a formas diferentes a las conocidas en el mundo. El único espécimen del Valanginiense determinado, la tortuga Notoemys zapatocaensis muestra su mayor afinidad con una forma de finales del Jurásico de Argentina (Cadena y Gaffney, 2005). Los elasmosaurios y los pliosauroideos del Barremienseo y Aptiense colombianos son morfológicamente más próximos a las formas contemporáneas australianas y a las norteamericanas posteriores (turonienses) (Welles, 1962; Hampe, 1992). Para los ictiosaurios del Barremiense-Aptiense las formas colombianas estudiadas hasta ahora no muestran afinidades marcadas con las formas contemporáneas; solo se puede mencionar la mayor proximidad morfológica craneana de $P$. sachicarum con la forma norteamericana Platypterygius americanus de edad posterior (Albiense-Cenomaniense) (Páramo-Fonseca, 1997a). Los mosasaurios colombianos muestran una cercana afinidad con las formas que habitaron en el mar interior de Norteamérica desde el Turoniense hasta el Campaniense (Polcyn y Bell, 2005; Páramo-Fonseca, 2013). Así, de manera general las formas presentes en Colombia muestran para el Cretácico Inferior una mayor afinidad con formas del hemisferio sur, mientras que las del Cretácico Superior lo hacen con formas del hemisferio norte. 


\section{CONCLUSIONES}

La revisión que se presenta aquí indica que los restos de reptiles marinos son abundantes en Colombia y que su estudio es aún limitado. Las continuas manifestaciones de fósiles de esqueletos y restos craneanos bien preservados han despertado paulatinamente el interés de los investigadores nacionales, quienes en las dos últimas décadas se han vinculado activamente a la labor de exploración, preparación, descripción y estudio de estos restos. En los últimos años ha crecido la vinculación de estudiantes al estudio de los reptiles marinos, lo cual asegura un desarrollo incremental de su conocimiento. Este alentador panorama muestra que la paleontología de los reptiles marinos en Colombia está actualmente consolidando sus bases.

El conocimiento alcanzado hasta ahora permite obtener un primer panorama de la historia de los reptiles marinos que habitaron en el mar cretácico de Colombia. Las características de las ocurrencias en las capas del Cretácico sugieren que las condiciones del mar que inundó la parte occidental de Colombia durante este período fueron favorables para el desarrollo y permanencia de distintos grupos de reptiles marinos y para la preservación de sus restos. Estas condiciones dejaron una gran riqueza fósil de restos de reptiles marinos que se manifiesta a lo largo de la Cordillera Oriental de Colombia. Los registros más antiguos corresponden a restos de tortugas, ictiosaurios y plesiosauroideos de edad Valanginiense. Las primeras se registran solo hasta el Aptiense, mientras que los segundos lo hacen hasta el Albiense y los últimos hasta el Turoniense. Los pliosauroideos por su parte se restringen al lapso Barremiense-Aptiense. En Villa de Leiva se registra un cambio en la abundancia relativa de especímenes de los distintos grupos, entre el piso Barremiense y el Aptiense, lo cual sugiere cambios en las condiciones paleoecológicas para ese lapso de tiempo. Finalmente los mosasaurios muestran una amplia permanencia durante el Cretácico Superior.

La buena preservación, la ubicación en el extremo noroccidental de Suramérica y la distribución estratigráfica de los fósiles de reptiles marinos de Colombia constituyen un invaluable potencial de información para el conocimiento de la paleo-herpetofauna marina que habitó durante el Cretácico en los mares del mundo.

\section{REFERENCIAS}

Acosta, C., Huertas, G. y Ruíz, P. 1979. Noticia preliminar sobre el hallazgo de un presunto Kronosaurus (Reptilia: Dolichorhynchopidae) en el Aptiano Superior de Villa de Leyva, Colombia. Lozanía, Acta Zoológica Colombiana 28: 1-7.

Bardet, N. 1992. Stratigraphic evidence for the extinction of the ichthyosaurs. Terra Nova 4: 649-656.

Bardet, N., Pereda Suberbiola, X. y Jalil, N.E. 2003. A new mosasauroid (Squamata) from the Late Cretaceous (Turonian) of Morocco. Comptes Rendus Palevol 2: 607-616.

Baur, G. 1887. On the morphology and origin of the Ichthyopterygia. American Naturalist 21: 837-840.

Baur, G. 1893. Notes on the classification of the Cryptodira. American Naturalist 27: 672-675.

Botero-Restrepo, G. 1945. [Localidad fosilifera de vertebrados e invertebrados en Leiva, Departamento de Boyacá. Informe del Servicio Geológico Nacional, No. 495, Bogotá, 7 p. Inédito.].

Bräm, H. 1965. Die Schildkröten aus dem oberen Jura (Malm) der Gegend von Solothurn. Schweizerische Palaöntologische Abhandlungen 83: 1-190.

Bürgl, H. 1954. El Cretácico Inferior en los alrededores de Villa de Leiva, Boyacá. Boletín Geológico del Instituto Geológico Nacional 2: 5-22.

Cadena, E.A. 2011a. Potential earliest record of Podocnemidoid Turtles from the Early Cretaceous (Valanginian) of Colombia. Journal of Paleontology 85: 877-881.

Cadena, E.A. 2011b. First record of eucryptodiran turtles from the Early Cretaceous (Valanginian), at the northernmost part of South America. South American Journal of Herpetology 6: 49-53.

Cadena, E.A. y Gaffney, E.S. 2005. Notoemys zapatocaensis, a new side-necked turtle (Pleurodira: Platychelyidae) from the Early Cretaceous of Colombia. American Museum Novitates 3470: 1-19.

Cadena, E.A. y Parham, J.F. 2015. Oldest known marine turtle? A new protostegid from the Lower Cretaceous of Colombia. $\mathrm{Pa}$ leoBios 32: 1-42.

Cadena, E.A., Jaramillo, C.A. y Bloch, J.I. 2013. New material of the Platychelyid turtle Notoemys zapatocaensis from the Early Cretaceous of Colombia; implications for understanding Pleurodira evolution. En: D.B. Brinkman, P.A. Holroyd y J.D. Gardner (Eds.), Morphology and evolution of turtles, Vertebrate Paleobiology and Paleoanthropology. Springer Science+Business Media, Dordrecht, p. 105-120.

Caldwell, M.W. y Palci, A. 2007. A new basal mosasauroid from the Cenomanian ( $U$. Cretaceous) of Slovenia with a review of mosasauroid phylogehy and evolution. Journal of Vertebrate Paleontology 27: 863-890.

Camp, C.L. 1923. Classification of the lizards. Bulletin of the American Museum of Natural History 48: 289-481.

Caracol Radio, 2015. Hallan nuevo fósil en Villa de Leyva. Caracol Radio, 15 de abril 2015. World Wide Web: http://caracol.com.co/ radio/2015/04/15/regional/1429068300_718487.html

Carpenter, K. 1999. Revision of North American elasmosaurs from the Cretaceous of the Western Interior. Paludicola 2: 148-173.

Cope, E.D. 1864. On the limits and relations of the Raniformes. Proceedings of the Academy of Natural Sciences of Philadelphia 16: 181-183.

Cope, E.D. 1868. On the origin of genera. Proceedings of the Academy of Natural Sciences of Philadelphia 20: 242-300.

Cope, E.D. 1869. Synopsis of the extinct Batrachia, Reptilia and Aves of North America, Part 1. Transactions of the American Philosophical Society (new series) 14: 1-252. 
Cope, E.D. 1872. A description of the genus Protostega, a form of extinct Testudinata. Proceedings of the American Philosophical Society 12: 422-433.

de Blainville, H.D. 1835. Description de quelques espèces de reptiles de la Californie, précédée de l'analyse d'un système général d'érpetologie et d'amphibiologie. Nouvelles annales $d u$ Muséum d'Histoire naturelle 4: 233-296.

de la Fuente, M. y Goñi, R. 1983. Primeras tortugas cretácicas marinas de Colombia. Geología Norandina 7: 43-48.

Etayo-Serna, F. 1968. El Sistema Cretácico en la región de Villa de Leyva y zonas próximas. Geología Colombiana 5: 14-25.

Etayo-Serna, F. 1979. Zonation of the Cretaceous of central Colombia by ammonites. Publicaciones Geológicas Especiales del INGEOMINAS 2: 1-186.

Etayo-Serna, F. 1994. Epílogo: A modo de Historia Geológica del Cretácico en el Valle Superior del Magdalena. En: Etayo-Serna (Ed.), Estudios Geológicos del Valle Superior del Magdalena. Publicación Ecopetrol-Universidad Nacional de Colombia, W. Taller Editorial LTDA, Bogotá, p. 1-5.

Etayo-Serna, F., Renzoni, G. y Barrero, D. 1976. Contornos Sucesivos del Mar Cretáceo en Colombia. Primer Congreso colombiano de Geología, Memorias: 217-252.

Forero, O.H. y Sarmiento, R.L.F. 1985. La facies evaporítica de la Formación Paja en la región de Villa de Leiva. En: F. Etayo-Serna y F. Lavede (Eds.), Proyecto Cretácico. Publicaciones Geológicas Especiales del INGEOMINAS, 16, Bogotá, p. 1-16.

Gaffney, E.S. 1975. A phylogeny and classification of the higher categories of turtles. Bulletin of the American Museum of Natural History 155: 389-436.

Gervais, P. 1853. Observations relatives aux reptiles fossiles de France. Comptes Rendus de l'Académie des Sciences de Paris 36: 374-377, 470-474.

Gómez, J.C. 2013. [Estudio de Restos Fósiles de Pliosaurio del Cretácico Inferior de Villa de Leiva. Trabajo de grado, Departamento de Geociencias, Facultad de Ciencias, Universidad Nacional de Colombia, 21 p. Inédita.].

Gómez-Pérez, M. 2001. [Estudio morfológico y taxonómico del cráneo de un reptil marino proveniente de capas de la Formación Paja, quebrada Pavachoque, municipio de Sutamarchán. Tesis de pregrado, Departamento de Geociencias, Facultad de Ciencias, Universidad Nacional de Colombia, 132 p. Inédita.].

Gómez-Pérez, M. 2008. [The palaeobiology of an exceptionally preserved colombian pliosaur (Sauropterygia: Plesiosauria). Ph.D. dissertation, University of Cambridge, Neunman College, Cambridge, 243 p. Inédita.].

Gómez-Pérez, M. y Noè, L. 2009. A pliosaur from the Lower Cretaceous of Colombia: the implications of a new genus. Journal of Vertebrate Paleontology 29, Supplement Abstracts of Papers 3: $107 \mathrm{~A}$.

Goñi, R. y Gasparini, Z.B. 1983. Nuevos restos de 'Alzadasaurus colombiensi' (Reptilia, Plesiosauria) del Cretácico temprano de Colombia. Geología Norandina 7: 49-54.

Gray, J.E. 1825. A synopsis of the genera of reptiles and amphibia, with a description of some new species. Annals of Philosophy, New Series 10: 193-217.

Guzmán, G. 1985. Los Grifeidos infracretácicos Aetostreon couloni y Ceratrostreon boussingaulti, de la Formación Rosablanca como indicadores de oscilaciones marinas. En: F. Etayo-Serna y F. Lavede (Eds.), Proyecto Cretácico. Publicaciones Geológicas Especiales del INGEOMINAS, 16, Bogotá, 1-16.

Hampe, O. 1992. Ein großwüchsiger Pliosauride (Reptilia: Plesiosauria) aus der Unterkreide (oberes Aptium) von Kolumbien.
Courier Forschungsinstitut Senckenberg, Frankfurt am Main 145: $1-32$.

Hampe, O. 2005. Considerations on a Brachauchenius skeleton (Pliosauroidea) from the lower Paja Formation (late Barremian) of Villa de Leyva area (Colombia). MitteMuseum für Naturkunde 8: 37-51.

Hernández-Camacho, J. y De Porta, J. 1963. El primer ictiosaurio del Cretácico de Colombia. Boletín de Geología, Universidad Industrial de Santander 12: 77-83.

Hubach, E. 1931. [Geología petrolifera del departamento de Norte de Santander. Informe del Servicio Geológico Nacional, No. 176, parte A, Bogotá, 218 p. Inédito.].

Hubach, E. 1942. [El plesiosaurio de Leiva, Departamento de Boyacá. Informe del Servicio Geológico Nacional, No. 852, Bogotá, 7 p. Inédito.].

Huertas, G. 1971. Theobroma verum. Mutisia Acta Botánica Colombiana 34: 1-10.

Konishi, T. y Caldwell, M.W. 2011. Two new plioplatecarpine (Squamata, Mosasauridae) genera from the Upper Cretaceous of North America, and global phylogenetic analysis of plioplatecarpines. Journal of Vertebrate Paleontology 31: 754-783.

McGowan, C. 1972. The systematics of Cretaceous ichthyosaurs with particular reference to the material from North America. Contributions to Geology 11: 9-29.

Moreno, C. 2010. Descubren fósil prehistórico de depredador marino. UN Periódico, 13 de marzo 2010. World Wide Web: http://www.unperiodico.unal.edu.co/dper/article/

Nicholls, E.L. y Meckert, D. 2002. Marine reptiles from the Nanaimo Group (Upper Cretaceous) of Vancouver Island. Canadian Journal of Earth Sciences 39: 1591-1603.

Padilla, C. y Parra, M. 2009. Acid preparation of fossils using sulfamic acid, a weak organic acid, and its advantages over acetic and formic acid preparation. Journal of Vertebrate Paleontology 29, Supplement Abstracts of Papers 3: 160A.

Padilla, C.B., Páramo, M.E., Noè, L., Gómez, M. y Parra, M.L. 2010. Acid preparation of large vertebrate specimens. Geological Curators Group 9: 213-220.

Palci, A., Caldwell, M.W. y Papazzoni, C.A. 2013. A new genus and subfamily of mosasaurs from the Upper Cretaceous of Northern Italy. Journal of Vertebrate Paleontology 33: 599- 612.

Páramo, M.E. 1991. [Posición sistemática de un reptil marino con base en los restos fósiles encontrados en capas del Cretácico superior en Yaguará (Huila). Tesis de pregrado, Departamento de Geociencias, Facultad de Ciencias, Universidad Nacional de Colombia. 112 p. Inédita.].

Páramo, M.E. 1994. Posición sistemática de un reptil marino con base en los restos fósiles encontrados en capas del Cretácico superior en Yaguará Huila. Revista de La Academia Colombiana de Ciencias Exactas Físicas y Naturales 72: 63-80.

Páramo-Fonseca, M.E. 1997a. Platypterygius sachicarum (Reptilia, Ichthyosauria) nueva especie del Cretácico de Colombia. Revista Ingeominas 6: 1-12.

Páramo-Fonseca, M.E. 1997b. [Les vertébrés marins du Turonien de la Vallée Supérieure du Magdalena, Colombie, systématique, paléoécologie et paléobiogéographie. Thèse de Doctorat, Université de Poitiers, Poitiers, 174 p., 25 pls. Inédita.].

Páramo-Fonseca, M.E. 2000. Yaguarasaurus columbianus (Reptilia, Mosasauridae), a primitive mosasaur from Turonian (Upper Cretaceous) of Colombia. Historical Biology 14: 121-131.

Páramo-Fonseca, M.E. 2012. Mosasauroids from Colombia. Bulletin de la Société Géologique de France 183: 103-109.

Páramo-Fonseca, M.E. 2013. Eonatator coellensis nov. sp. (Squa- 
mata: Mosasauridae), a new species from the Upper Cretaceous of Colombia. Revista de La Academia Colombiana de Ciencias Exactas Físicas y Naturales 37: 499-518.

Páramo, M., Etayo-Serna, F., Gómez-Pérez, M., Padilla, C. y Noè, L. 2009. New marine reptiles from the Cretaceous of Central Colombia. Journal of Vertebrate Paleontology 29, Supplement Abstracts of Papers 3: $161 \mathrm{~A}$.

Polcyn, M.J. y Bell, G.L. Jr. 2005. Russellosaurus coheni n. gen., n. sp., a 92 million-year-old mosasaur from Texas (U.S.A.), and the definition of the parafamily Russellosaurina. Netherlands Journal of Geosciences 84: 321-333.

Posada-Swafford, A. 2014. Colombia devela un impresionante depósito de fósiles marinos de hace más de 100 millones de años. Scientific American Español, 16 de octubre 2014. World Wide Web: http://www.scienticamerican.com/español/noticias/

Royo y Gómez, J. 1943. Rocas y fósiles del Cretácico de la región de Ubaté (Norte de Cundinamarca). Informe del Servicio Geológico Nacional, No. 326. En: Edición del Servicio Geológico Nacional (Ed.), Compilación de los estudios geológicos oficiales en Colombia, Tomo 22. Bogotá, p. 115-120.

Seeley, H.G. 1874. Note on some of the generic modifications of the plesiosaurian pectoral arch. Quarterly Journal of the Geological Society of London 30: 436-449.

Smith, D.T.J. 1989. [The cranial morphology of fossil and living sea turtles (Cheloniidae, Dermocheliyidae and Desmatochelyidae). Ph.D. Dissertation, School of Geological Sciences Kingston Polytechnic, Surrey, England, 310 p. Inédita.].
UCMP. 2015. Fossil Collections. En: UCMP Editors, University of California Museum of Paleontology. World Wide Web: http://ucmpdb.berkeley.edu/cgi/ucmp

Welles, S.P. 1943. Elasmosaurid plesiosaurs with description of new material from California and Colorado. University of California, Memoirs 13: 125-254.

Welles, S.P. 1962. A new species of elasmosaur from the Aptian of Colombia and a review of the Cretaceous plesiosaurs. University of California Publications in the Geological Sciences 44: 1-96.

Williston, S.W. 1894. A new turtle from the Benton Cretaceous. Kansas University Quarterly 3: 5-18.

Zárate, G. 2006. En Villa de Leyva desentierran un gran fósil marino. El Tiempo, 16 de enero 2006. World Wide Web: http://www.eltiempo.com/archivo/documento/MAM-1885041

Recibido: 12 de junio 2015

Aceptado: 19 de octubre 2015 